\title{
The Summarized Weighted Mean of Maxima Defuzzification and Its Application at the End of the Risk Assessment Process
}

\author{
Tamás Portik ${ }^{1}$ and László Pokorádi ${ }^{2}$ \\ ${ }^{1}$ University of Debrecen, Faculty of Engineering, Ótemető utca 2-4, Debrecen, \\ Hungary, portik@eng.unideb.hu \\ ${ }^{2}$ Óbuda University, Donát Bánki Faculty of Mechanical and Safety Engineering, \\ Népszinház u. 8, Budapest Hungary, e-mail: pokoradi.laszlo@bgk.uni-obuda.hu
}

\begin{abstract}
Nowadays, it is indispensible to take into consider the risk in modern technical management especially in hydraulic systems under different circumstances. The reliability of a hydraulic system is a well investigated area by researchers because of system damages or crash down can cause cost losses, human injuries or death. If the hydraulic system gives back imprecise or vague data and the reports of expert given by linguistic variables during the inspection then the risk assessment must be calculated with fuzzy mathematics. In this paper, the authors propose a modified fuzzy rule based risk assessment method for the risk assessment of hydraulic systems. The difference between the proposed and the original methods is the defuzzification sub process only. This defuzzification sub process is called Summarized Defuzzification (SDF).
\end{abstract}

Keywords: hydraulic system, fuzzy risk assessment, Summarized Defuzzification

\section{Introduction}

Pokorádi had written a paper about fuzzy rule based hydraulic risk assessments [13] in which some questions are raised. Before fuzzyfying, the averaging of opinions of experts does not reflect the full spectrum of expert opinions. Therefore authors work out a modified fuzzy rule based risk assessment method to handle extreme opinions on input data because of statistical averaging skips them immediately. The motivation was to handle these extreme opinions in another way because they are opinions from experts so they contain information from the investigated area even if experts do not have longtime experiences. On the other hand if one does not have enough data, moreover they are imprecise and uncertainty, from questionnaires then the statistical averaging is not the best way to handle extreme opinions. The expert opinions, knowledge are mainly reported in linguistic variables. 
The first publication is written by Zadeh in [20], where he introduced the notion of fuzzy sets. To have a good introduction into fuzzy set and logic theory, the authors recommend the Ross' book [15] specialized for engineers. To measure imprecise and uncertainty data and notions one must use fuzzy set and logic theory which can handle the linguistic variables and logically inconsistent statements [15].

Fuzzy risk matrix is a very useful tool for semi-quantitative risk assessment which is provided by Markowski \& Mannan to handle process activities of hazardous materials [7]. Therefore they developed low-cost, standard and high-cost matrixes.

In Markowski et. al.' paper, they introduced the risk correction index to take into consideration uncertainty concerned with the identification of representative accident scenario which provided more realistic results [8].

Shi et. al.'s analyzed results interpret the effectiveness of the Gaussian-mixed fuzzy clustering model on valence-arousal-related fMRI data-set for feature exracting operations including power spectrum density, spline, shape-preserving and cubic fitting methods [16].

The authors cite another application of fuzzy set and logic theory like Johanyák shows the clonal selection algorithm for tuning up the fuzzy inference system which was tested in case of SISO and MISO systems for which it is successfully usable [4].

Nagy et. al. proposed a stochastic approach for fuzzy control which is extremely fast, robust and simulation of the inverse pendulum makes validate it [9].

Rezaei et. al. proposed analytic hierarchy process with application for fuzzy multicriteria decision making analysis which takes into consideration more important factors in decision making if it has fuzzy enviroment [14].

Singh \& Markaset' paper represents a risk based inspection planning for oil and gas pipe systems based on fuzzy framework [17]. The inspected rate of corrosion and the efficiency of inspection are taken into consideration as fuzzy variables to calculate Trust in Inspection Results and Trust in Predicted Results which are combined together for estimated corrosion for carbon steel pipes.

Liu et. al. have written a comprehensive literature review about computing with words using fuzzy mathematics [6]. They survey the papers for ordinal linguistic approach, fuzzy rule based, fuzzy number and fuzzy extension of typical probabilistic risk assessment and some miscellaneous applications.

Cai represents a literature review about failure-oriented view to holistic view of system failure engineering combined with fuzzy methodology [1]. In his paper, there are shown a lot of engineering case-studies and fuzzy mathematics problems raised by applications.

Fuzzy risk analysis problems are proposed to solve with arithmetic operator which satisfy all properties for trapezoidal fuzzy numbers according to $\mathrm{Xu}$ et. al. 
[18] - and investigated the similarity of the trapezoidal fuzzy numbers. Moreover they made an experiment with 30 sets of fuzzy trapezoidal number for calculation risk which results are acceptable because they are close to human thinking.

Accuracy of agricultural natural disaster risk assessment is improved, which work efficiency is about $28 \%$ higher then the histogram method, with developed information distribution and interior-outer-set model for calculating fuzzy probability distribution to show imprecision by Huang in [2].

Jamshidi et. al. designed the Mamdani algorithm for pipeline risk assessment based on fuzzy logic toolbox of MATLAB to use one of the most popular techniques in pipeline risk assessment namely relative risk score methodology [3]. Moreover, they made a comparison between the traditional risk assessment and the fuzzy methodology based risk assessment on a case-study in which the last one provides more accurate and precise results.

Li et. al. proposed the fuzzy human error risk assessment for determining human error risk importance as function of human error probability, error-effect probability and error consequence severity and they provide the Mamdani techniques in fuzzy toolbox of MATLAB [5]. The results of case-studies are more realistic, practicable and valuable.

Wang et. al. proposed fuzzy failure mode and effect analysis with weighted geometric mean for prioritization of failure modes by fuzzy risk priority numbers [19]. The alpha-level sets centroid defuzzification method is used for ranking the failure modes.

Pokorádi wrote about the reliability and fuzzy rule based risk assessment with an application in a special helicopter mission for reliability and risk in [10]. Pokorádi's book [12] speaks about system and process modeling in engineering which contains introduction in fuzzy set and logic theory and its applications specialized for engineers like fuzzy decision-making and fuzzy failure mode and effect analysis. Pokorádi has applied the fuzzy rule based risk assessment in military science in [10].

All of cited papers above show how any kind of fuzzy risk assessment is important on a lot of part of science like engineering, informatics, agriculture and so on.

The general aim of the authors is to work out new fuzzy rule based risk assesment methods which consists new defuzzification where the opinion of experts goes through the process till the composition part during the decision-making without averaging the experts' opinions at the beginning of the process. During suggested method averaging occurs at the end of the assessment process. Therefore the total spectrum of experts' opinions has more effect on results. Because of different intermediate results arise in inference and composition subprocesses in case of different experts' opinions. 
This means more carefully quantified experts' opinion as result which reflect more carefully the experts' opinions. The aim of this paper is to present a solution to handle extreme opinions without averaging at the beginning of fuzzy rule based risk assessment. This assessment process uses called Summarized DeFuzzification (SDF).

The rest part of the paper is organized as follows: Section 2 provides a short overview about fuzzy risk assessment and introduces the theoretical background of the SDF method. Section 3 shows an easy case-study about a hydraulic system for its risk computation. In the last section, authors give a summary of their work.

\section{Fuzzy Rule-based Risk Assessment}

In this section authors proved a short overview about traditional fuzzy rule-based risk assessment and they introduce the summarized defuzzification.

\subsection{Traditional Fuzzy Rule-based Risk Assessment}

The traditional fuzzy decision process has next parts: fuzzyfication, inference, composition and defuzzification (Figure 1). Sometimes it is joined together the composition and defuzzification process.

Fuzzyfication sub process means that the input data are fuzzyfied with predefined fuzzy membership functions for further computations.
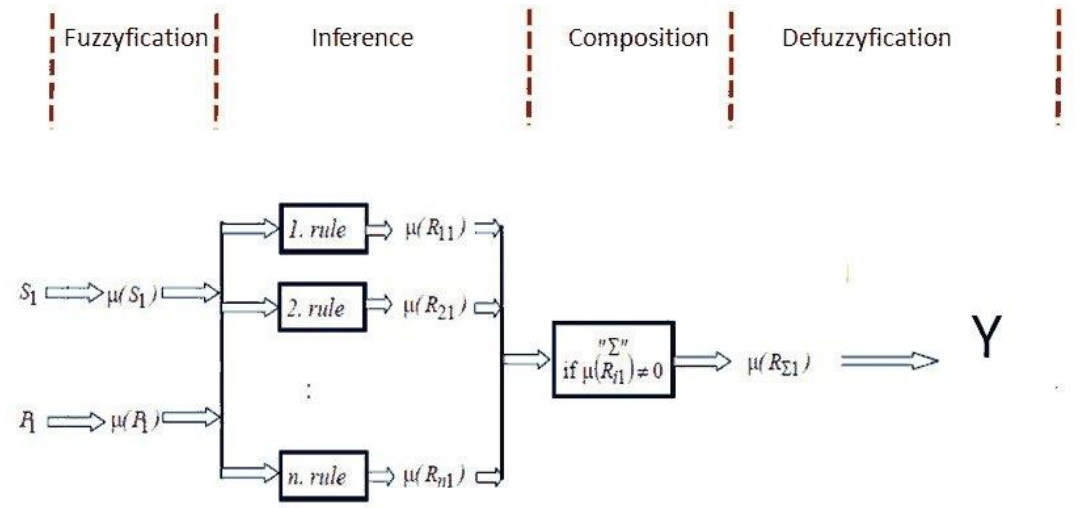

Figure 1

Traditional fuzzy process flow-chart (source: [12])

Inference sub process means if the predicates are joined together with logical AND then the data are combined together with t-norm. If the predicates are 
connected with logical OR then the data are handled with s-norm. The If-Then rules are represented in the Risk Assessment Matrix (RAM) for easy handling (like Figure 10).

Composition sub process means each category which has more values, is composed with s-norm - usually used maximum operator. One takes only the non zero inference into consider in composition sub process.

Defuzzification sub process means that the fuzzy result is converted in a crisp value. This is necessary especially in technical management because decision makers like managers, leaders can not handle the fuzzy results.

There is a lot of theoretical and practical method for defuzzification process. The authors choose the Weighted Mean of Maximum (WMM) - because of the "base" of case study [13] used that method - where of course it has many different types. In this paper it is defined by equation (1).

$$
R_{W M M}=\frac{\sum_{i=1}^{n} \mu_{i} \cdot z_{i}}{\sum_{i=1}^{n} \mu_{i}}
$$

n $\quad-$ the number of conclusions which are different from 0 ,

$\mu_{\mathrm{i}} \quad-$ the value of $\mathrm{i}$-th fuzzy membership function,

$\mathrm{Z}_{\mathrm{i}} \quad-$ the weighted value of the i-th fuzzy membership function.

The weighted value of fuzzy membership function means either the point on abscissa where fuzzy membership function takes its maximum value at only one point or the midpoint of the interval if this maximum value is on an interval. This method is common used in literatures i.e. $[12,15]$.

\subsection{Summarized Defuzzification (SDF)}

The fuzzy rule based risk assessment using Summarized Defuzzification (SDF) method is the next: one takes the traditional fuzzy process till the composition part, in other words one let the input opinions, where some of expert teams can have extreme opinion, through fuzzyfication, inference and composition sub process.

One collects all this fuzzy results. This means one has converted all extreme opinions to fuzzy risk assessment. Then one can defuzzyfy for all fuzzy risk opinions, if crisp value of risk is needed, where the SDF is shown on Figure 2. To get it clear, the definition of summarized defuzzification is in equation (2). 


$$
R_{S W M M}=\frac{\sum_{i=1}^{m} \sum_{j=1}^{n} \mu_{i j} \cdot z_{i j}}{\sum_{i=1}^{m} \sum_{j=1}^{n} \mu_{i j}}
$$

$$
\begin{aligned}
& \mathrm{R}_{\text {swмm }} \text { - Crisp value of summarized defuzzification, } \\
& \mathrm{m} \text { - the number of opinions (input data), } \\
& \text { n } \quad-\text { the number of fuzzy membership function, } \\
& \mu_{\mathrm{ij}} \quad-\text { the value of } \mathrm{j} \text {-th fuzzy membership function } \\
& \text { which belongs to i-th opinion (input data), } \\
& z_{i j} \quad-\text { the weighted value of the } \mathrm{i} \text {-th fuzzy membership function } \\
& \text { in case of the j-the opinion. }
\end{aligned}
$$

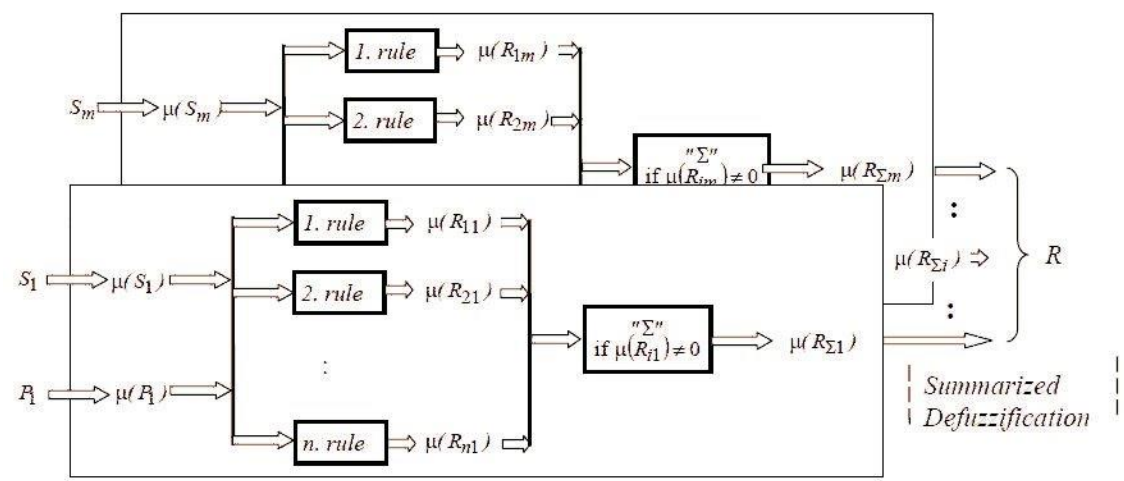

Figure 2

Summarized defuzzification flow-chart

One remark again: The weighted value of fuzzy membership function has either only one point where fuzzy membership function takes its maximum value or the midpoint of the interval if this maximum value is over an interval. This method can easily and quickly to be computed. The equation 2. express that the experts' opinion are taken into considaration at the end of defuzzification process. In this paper all computation is made by Maple in which a module is developed by authors. 


\section{Case-Study}

In this case-study the authors investigate an easy sample for a hydraulic system. This sample was investigated in an earlier paper with the traditional fuzzy rule based method which is written in [13] when Pokorádi used opinions of two expert teams that investigated a hydraulic system from different points of view. During the calculation of case-study, the authors use triangular and trapezoidal fuzzy membership functions on linear (equations (3)-(4) and Figures 3-4) and logarithmic scaled abscissa (equations (5)-(6) and Figures 5-6).

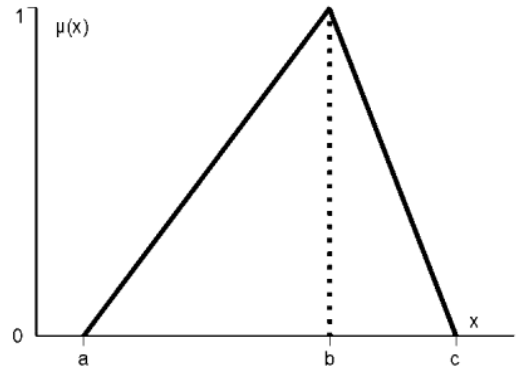

Figure 3

Triangular fuzzy membership function

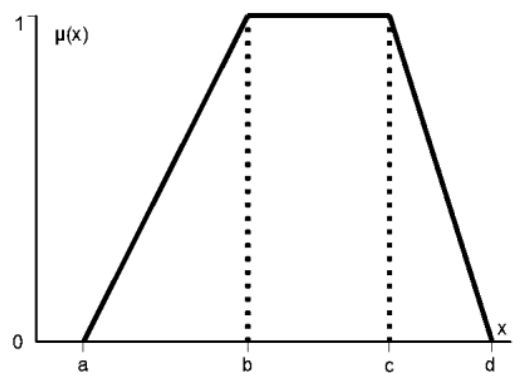

Figure 4

Trapezoidal fuzzy membership function

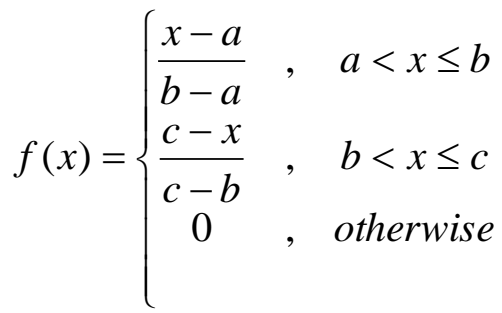

Sign $: f:(a, b, c)$. 


$$
f(x)=\left\{\begin{array}{ccc}
\frac{x-a}{b-a} & , & a<x \leq b \\
1 & , & b<x \leq c \\
\frac{d-x}{d-c} & , & c<x \leq d \\
0 & , & \text { otherwise }
\end{array}\right.
$$

Sign $: f:(a, b, c, d)$

$$
\varphi(x)=\left\{\begin{array}{ccc}
\frac{\lg (x)-\lg (a)}{\lg (b)-\lg (a)}, & a<x \leq b \\
\frac{\lg (c)-\lg (x)}{\lg (c)-\lg (b)} & , \quad b<x \leq c \\
0 & , & \text { otherwise }
\end{array}\right.
$$

Sign $: \varphi:(a, b, c)$.

Firstly the severity, probability, and risk fuzzy sets categories (Table 1, Figures 79) and the RAM (Figure 10) should be defined according to experts and their experiences and knowledge.

$$
\varphi(x)=\left\{\begin{array}{ccc}
\frac{\lg (x)-\lg (a)}{\lg (b)-\lg (a)} & , & a<x \leq b \\
1 & , & b<x \leq c \\
\frac{\lg (d)-\lg (x)}{\lg (d)-\lg (c)} & , & c<x \leq d \\
0 & , & \text { otherwise }
\end{array}\right.
$$

Sign : $\varphi:(a, b, c, d)$

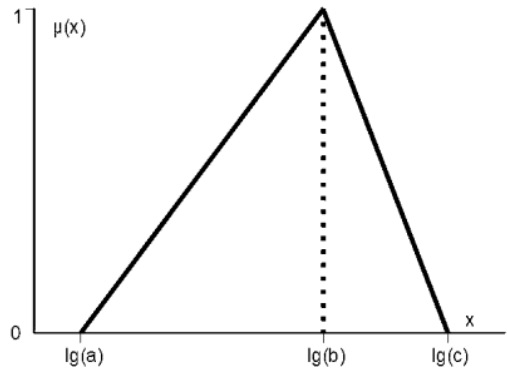

Figure 5

Triangular membership function in case of logarithmic scaled abscissa 


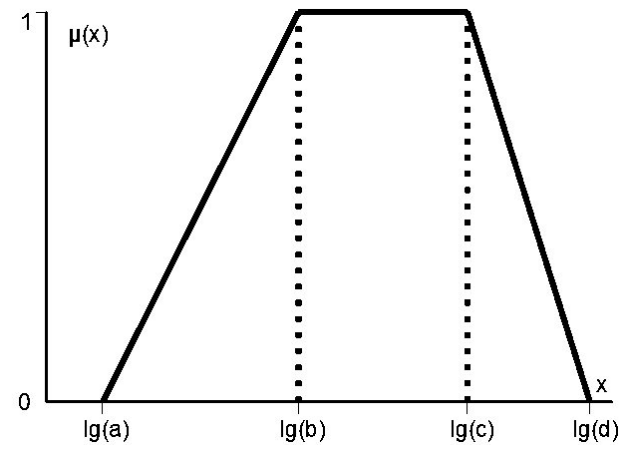

Figure 6

Trapezoidal membership function in case of logarithmic scaled abscissa

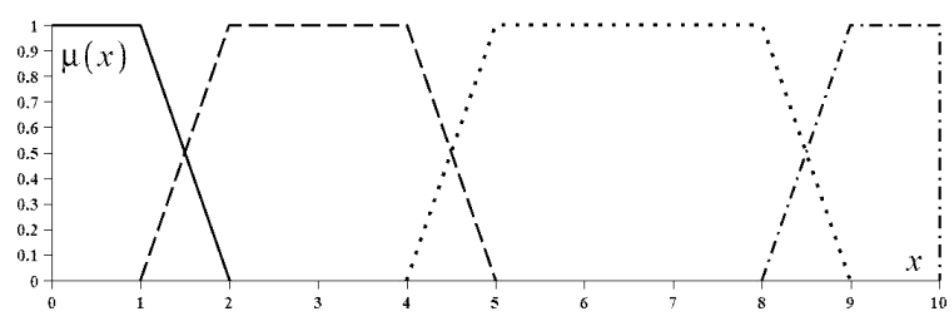

- Negligible $-\cdot$ Moderate $\cdots$ Critical $-\cdot$ Catastrophic

Figure 7

Fuzzy membership function of severity categories

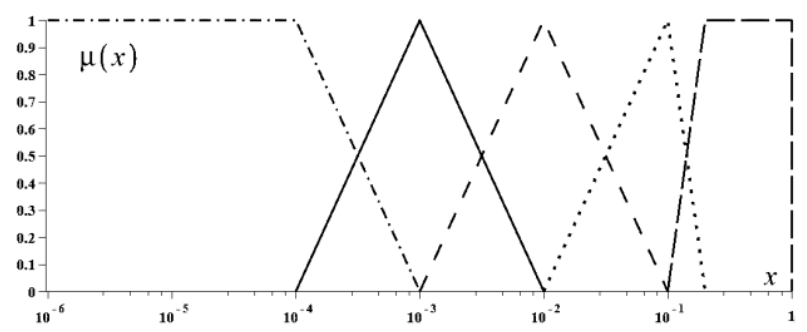

$-\cdot$ Unelikely - Seldom - Occasional $\cdots$ Likely - Frequent

Figure 8

Fuzzy membership function of probability categories 


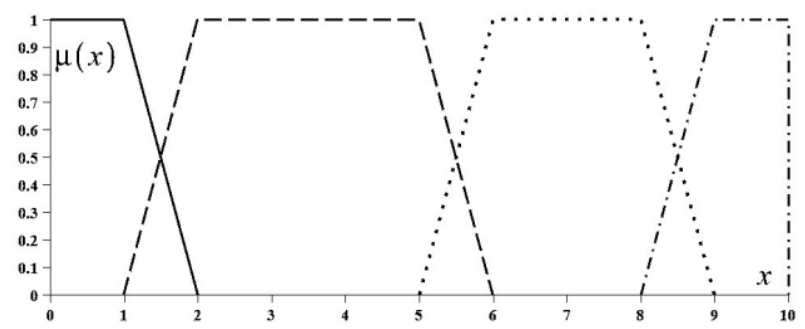

- Low $-\cdot$ Medium $\cdots$ High $-\cdot$ Extra High

Figure 9

Fuzzy membership function of risk categories

Table 1

The severity-, probability- and risk categories

\begin{tabular}{|l|l|l|}
\hline \multirow{5}{*}{ Severity } & Negligible & $\mu_{\text {Neg }}=f:(0,0,1,2)$ \\
\cline { 2 - 3 } & Moderate & $\mu_{\text {Mod }}=f:(1,2,4,5)$ \\
\cline { 2 - 3 } & Critical & $\mu_{\text {Crit }}=f:(4,5,8,9)$ \\
\cline { 2 - 3 } & Catastrophic & $\mu_{\text {Cat }}=f:(8,9,10,10)$ \\
\hline \multirow{5}{*}{ Probability } & Unlikely & $\mu_{\text {Unl }}=\varphi:\left(0,0,10^{-4}, 10^{-3}\right)$ \\
\cline { 2 - 3 } & Seldom & $\mu_{\text {Sel }}=\varphi:\left(10^{-4}, 10^{-3}, 10^{-2}\right)$ \\
\cline { 2 - 3 } & Occasional & $\mu_{\text {Occ }}=\varphi:\left(10^{-3}, 10^{-2}, 10^{-1}\right)$ \\
\cline { 2 - 3 } Risk & Likely & $\mu_{\text {Lik }}=\varphi:\left(10^{-2}, 10^{-1}, 0.2\right)$ \\
\cline { 2 - 3 } & Frequent & $\mu_{\text {Freq }}=\varphi:\left(10^{-1}, 0.2,1,1\right)$ \\
\hline \multirow{5}{*}{} & Low & $\mu_{\text {Low }}=f:(0,0,1,2)$ \\
\cline { 2 - 3 } & Medium & $\mu_{M e d}=f:(1,2,5,6)$ \\
\cline { 2 - 3 } & High & $\mu_{H}=f:(5,6,8,9)$ \\
\cline { 2 - 3 } & Extra High & $\mu_{E H}=f:(8,9,10,10)$ \\
\hline
\end{tabular}

Two types of hazard are investigated in our hydraulic system namely burst in return pipe and pump failure (Table 2). The results of traditional fuzzy process are in "traditional" columns. The data are gathered from investigated failure rate with statistical methods for probability and the scale is from 0 to 1 for it. The experts/engineers have been divided into two expert groups. Let them A and B. The opinion about severity, is given by team A, are 3.8 for burst return pipe and 8 
for pump failure on scale from 0 to 10 . The opinion about severity, is given by team B, are 4.6 for burst return pipe and 9 for pump failure again on scale from 0 to 10 . The averages are 4.2 for burst in return pipe and 8.5 for pump failure. The results of average input mean the traditional result which is published in [13].

\begin{tabular}{|c|c|c|c|c|c|}
\hline & Frequent & Likely & Occasional & Seldom & Unlikely \\
\hline Catastrophic & \multirow[t]{2}{*}{ EH } & & \multirow[t]{2}{*}{$\mathrm{H}$} & & M \\
\hline Critical & & & & $\mathbf{M}$ & \\
\hline Moderate & $\mathrm{H}$ & & $\mathbf{M}$ & \multirow{2}{*}{\multicolumn{2}{|c|}{ L }} \\
\hline Negligible & $\mathbf{M}$ & & & & \\
\hline
\end{tabular}

EH - Extra High; $\mathbf{H}-$ High; $\mathbf{M}-$ Medium; L - Low.

Figure 10

Risk Assessment Matrix (RAM) (source: [13])

The authors emphasize not to do averaging because of all expert has notable knowledge about the investigated hydraulic system even if some of them have not too much experiences.

Table 2

The results of traditional and summarized defuzzification

\begin{tabular}{|c|c|c|c|c|c|c|c|}
\hline \multirow{4}{*}{ 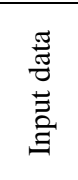 } & \multirow{2}{*}{ Name of Hazard } & \multicolumn{3}{|c|}{ Burst in return pipe } & \multicolumn{3}{|c|}{ Pump failure } \\
\hline & & Traditional & A & $\mathrm{B}$ & Traditional & A & B \\
\hline & Severity & 4.2 & 3.8 & 4.6 & 8.5 & 8 & 9 \\
\hline & Probability & \multicolumn{3}{|c|}{0.0002} & \multicolumn{3}{|c|}{0.005} \\
\hline \multirow{4}{*}{ 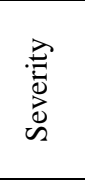 } & Catastrophic & 0 & 0 & 0 & 0.5 & 0 & 1 \\
\hline & Critical & 0.2 & 0 & 0.6 & 0.5 & 1 & 0 \\
\hline & Moderate & 0.8 & 1 & 0.4 & 0 & 0 & 0 \\
\hline & Negligible & 0 & 0 & 0 & 0 & 0 & 0 \\
\hline \multirow{5}{*}{ 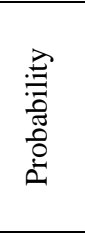 } & Frequent & \multicolumn{3}{|c|}{0} & \multicolumn{3}{|c|}{0} \\
\hline & Likely & \multicolumn{3}{|c|}{0} & \multicolumn{3}{|c|}{0} \\
\hline & Occasional & \multicolumn{3}{|c|}{0} & \multicolumn{3}{|c|}{0.699} \\
\hline & Seldom & \multicolumn{3}{|c|}{0.301} & \multicolumn{3}{|c|}{0.301} \\
\hline & Unlikely & \multicolumn{3}{|c|}{0.699} & \multicolumn{3}{|c|}{0} \\
\hline \multirow{4}{*}{ 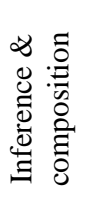 } & Extra high & 0 & 0 & 0 & 00 & 0 & 0 \\
\hline & High & 0 & 0 & 0 & 0.5 & 0.699 & 0.699 \\
\hline & Medium & 0.2 & 0 & 0.301 & 0.301 & 0.301 & 0 \\
\hline & Low & 0.699 & 0.699 & 0.6 & 0 & 0 & 0 \\
\hline \multicolumn{2}{|c|}{ Defuzzification } & 1.284 & \multicolumn{2}{|c|}{1.205} & 5.685 & \multicolumn{2}{|c|}{6.38} \\
\hline
\end{tabular}


The first step is fuzzyfying input data, this step can be seen for probability and severity on Table 2 . The severity and probability values are joined together with minimum operator according to risk assessment matrix (e.g. Table 3 for B group at burst in return pipe). If some risk categories have many values then they are joined together with maximum operator in composition part (e.g. Table 2) which is represented at Table 3. as well.

The fuzzy result is $\mathrm{L}-0.699$ after the calculation for opinion $\mathrm{A}$ at burst in return pipe. The fuzzy result is $\mathrm{M}-0.301$ and $\mathrm{L}-0.6$ after the calculation for opnion $\mathrm{B}$ at burst in return pipe. The fuzzy result is $\mathrm{L}-0.699$ after the calculation for opinion $\mathrm{A}$ at burst in return pipe. The fuzzy result is $\mathrm{M}-0.301$ and $\mathrm{L}-0.6$ after the calculation for opnion B at burst in return pipe.

Table 3

The RAM \& composition result

\begin{tabular}{|c|c|c|c|c|c|c|c|c|}
\hline & $\begin{array}{l}\text { Frequent } \\
0\end{array}$ & $\begin{array}{l}\text { Likely } \\
0\end{array}$ & $\begin{array}{l}\text { Occasional } \\
0\end{array}$ & \multicolumn{2}{|c|}{$\begin{array}{l}\text { Seldom } \\
0.301\end{array}$} & \multicolumn{2}{|c|}{$\begin{array}{l}\text { Unlikely } \\
0.699\end{array}$} \\
\hline \multirow{2}{*}{ Catastrophic } & A, 0 & \multirow{2}{*}{$\mathrm{EH}, 0$} & \multirow{2}{*}{$\mathrm{EH}, 0$} & \multirow{2}{*}{$\mathrm{H}, 0$} & \multirow{2}{*}{\multicolumn{2}{|c|}{$\mathrm{H}, 0$}} & \multirow{2}{*}{\multicolumn{2}{|c|}{ M, 0}} \\
\hline & $\mathrm{B}, 0$ & & & & & & & \\
\hline \multirow[b]{2}{*}{ Critical } & $\mathrm{A}, 0$ & \multirow[b]{2}{*}{$\mathrm{EH}, 0$} & \multirow[b]{2}{*}{$\mathrm{H}, 0$} & \multirow[b]{2}{*}{$\mathrm{H}, 0$} & \multirow[b]{2}{*}{ M } & 0 & \multirow[b]{2}{*}{$\mathrm{L}$} & 0 \\
\hline & B, 0.6 & & & & & $\begin{array}{l}0.30 \\
1\end{array}$ & & 0.6 \\
\hline \multirow[b]{2}{*}{ Moderate } & A, 1 & \multirow[b]{2}{*}{$\mathrm{H}, 0$} & \multirow[b]{2}{*}{$\mathrm{M}, 0$} & \multirow[b]{2}{*}{ M, 0} & \multirow[b]{2}{*}{$\mathrm{L}$} & $\begin{array}{ll}0.30 \\
1\end{array}$ & \multirow[b]{2}{*}{$\mathrm{L}$} & 0.699 \\
\hline & B, 0.4 & & & & & $\begin{array}{l}0.30 \\
1\end{array}$ & & 0.4 \\
\hline \multirow{2}{*}{ Negligable } & $\mathrm{A}, 0$ & \multirow{2}{*}{ M, 0} & \multirow{2}{*}{$\mathrm{L}, 0$} & \multirow[t]{2}{*}{$\mathrm{L}, 0$} & \multirow{2}{*}{\multicolumn{2}{|c|}{$\mathrm{L}, 0$}} & \multirow{2}{*}{\multicolumn{2}{|c|}{$\mathrm{L}, 0$}} \\
\hline & $\mathrm{B}, 0$ & & & & & & & \\
\hline \multicolumn{9}{|c|}{$\begin{array}{l}\text { After composition A opinion about risk for burst in return pipe: } \mathrm{L}-0.699 \\
\text { After composition } \mathrm{B} \text { opinion about risk for burst in return pipe: } \mathrm{M}-0.301, \mathrm{~L}-0.699\end{array}$} \\
\hline
\end{tabular}

To do SDF one must use equation (2). The number of opinions $n$ is 2, the number of fuzzy membership function at risk categories are $m=4$. So using the equation (2) one gets the result of the fuzzy opinion of experts for crisp value of risk 1.205 on a scale from 0 to 10 by burst in return pipe. At pump failure one gets the result for crisp value of risk 6.38. Both of results are different with SDF from the traditional fuzzy based computation.

After using of modified risk assessment, the Authors showed its complete results (not only these hazards shown above) to participators. Their general opinions were, the modified results are more carefully quantified of joint expertise according to the rate of experts' group. But the participators could not estimate the difference by a numerical crisp value. 


\section{Summary}

In this paper, authors have proposed a new point of view for handling extreme experts' opinions during fuzzy rule based risk assessment. This method uses summarized defuzzification which gives better crisp values for risk in risk assessment like traditional method according to Pokorádi. Of course like every risk predictions which are used in decision-making process, have been provided responsibilities of managers or leaders. The future aims to work out other SDF methods and to use them in general practice for technical management.

\section{References}

[1] K.-Y. Cai, System Failure Engineering and Fuzzy Methodology An Introductory Overview, Fuzzy Sets and Systems, Vol. 83, Issue 2, 8 October 1996, p. 113-133

[2] C. Huang, An Application of Calculated Fuzzy Risk, Information Sciences, Vol. 142, Issues 1-4, May 2002, pp. 37-56

[3] A. Jamshidi, A. Yazdani-Chamzini, S. H. Yakhchali, S. Khaleghi, Developing a New Fuzzy Inference System for Pipeline Risk Assessment, Journal of Loss Prevention in the Process Industries, Vol. 26, Issue 1, January 2013, pp. 197-208

[4] Zs. Cs. Johanyák, Clonal Selection-based Parameter Optimization for Sparse Fuzzy Systems, in Proc. of IEEE $16^{\text {th }}$ International Conference on Intelligent Engineering Systems (INES 2012), June 13-15, 2012, Lisbon, Portugal, pp. 369-373

[5] P.-C. Li, G.-H. Chen, L.-C. Dai, Z. Li, Fuzzy Logic-based Approach for Identifying the Risk Importance of Human Error, Safety Science, Vol. 48, Issue 7, August 2010, pp. 902-913

[6] J. L. Liu, H. Martinez, R. M. Rodríguez, V. Novozhilov, Computing with Words in Risk Assessment, International Journal of Computational Intelligence Systems, Vol. 3, Issue 4. pp. 396-419 (2010)

[7] A. S. Markowski, M. S. Mannan, Fuzzy Risk Matrix, Journal of Hazardous Materials, Vol. 159, Issue 1, 15 November 2008, pp. 152-157

[8] A. S. Markowski, M. S. Mannan, A. Kotynia (Bigoszewska), D. Siuta, Uncertainty Aspects in Process Safety Analysis, Journal of Loss Prevention in the Process Industries, Vol. 23, Issue 3, May 2010, pp. 446-454

[9] K. Nagy, Sz. Divéki, P. Odry, M. Sokola,V. Vujičić, A Stochastic Approach to Fuzzy Control, Acta Polytechnica Hungarica, Vol. 9, No. 6, (2012) pp. 29-48

[10] L. Pokorádi, Fuzzy Logic-based Risk Assessment, AARMS, Academic and Applied Research in Military Science, Volume 1, Issue 1 (2002) pp. 63-73

[11] L. Pokorádi, Application of Fuzzy Set Theory for Risk Assessment, Journal 
of KONBiN, No 2,3 (14, 15) 2010, Warsaw, pp. 195-204

[12] L. Pokorádi, Rendszerek és folyamatok modellezése, Campus Kiadó, Debrecen, Hungary (2008)

[13] L. Pokorádi, Risk Assessment based upon Fuzzy Set Theory, $15^{\text {th }}$ "Building Services Mechanical and Industrial Days" International Conference, 15-16 October 2009, Debrecen, Hungary, pp. 311-318

[14] P. Rezaei, K. Rezaie, S. Nazari-Shirkouhi, M. R. J. Tajabadi, Application of Fuzzy Multi-Criteria Decision Making Analysis for Evaluating and Selecting the Best Location for Construction of Underground Dam, Acta Polytechnica Hungarica, Vol. 10, No. 7 (2013) pp. 187-205

[15] T. J. Ross, Fuzzy Logic with Engineering Applications, John Wiley \& Sons Ltd., Singapore (2010)

[16] F. Shi, P. McC. Bush, A Gaussian-mixed Fuzzy Clustering Model on Valence-Arousal-related fMRI Data-Set, Acta Polytechnica Hungarica, Vol. 10, No. 8 (2013) pp. 85-104

[17] M. Singh, T. Markeset, A Methodology for Risk-based Inspection Planning of oil and Gas Pipes Based on Fuzzy Logic Framework, Engineering Failure Analysis, Vol. 16, Issue 7, October 2009, pp. 2098-2113

[18] Z. Xu, S. Shang, W. Qian, W. Shu, A Method for Fuzzy Risk Analysis Based on the New Similarity of Trapezoidal Fuzzy Numbers, Expert Systems with Applications, Vol. 37, Issue 3, 15 March 2010, pp. 19201927

[19] Y.-M. Wang, K.-S. Chin, G. K .K. Poon, J.-B. Yang, Risk Evaluation in Failure Mode and Effects Analysis using Fuzzy Weighted Geometric Mean, Expert Systems with Applications, Vol. 36, Issue 2, Part 1, March 2009, pp. 1195-1207

[20] L. Zadeh, Fuzzy Sets, Information and Control, Vol. 8 (1965) pp. 338-353 